\title{
Safety Profile of Ceftazidime-Avibactam: Pooled Data from the Adult Phase II and Phase III Clinical Trial Programme
}

\author{
Karen Cheng ${ }^{1} \cdot$ Paul Newell $^{2} \cdot$ Joseph W. Chow ${ }^{3} \cdot$ Helen Broadhurst $^{2} \cdot$ David Wilson $^{2} \cdot$ Katrina Yates $^{2}$. \\ Angela Wardman ${ }^{2}$
}

Published online: 29 June 2020

(c) Pfizer Inc. 2020

\begin{abstract}
Introduction Ceftazidime-avibactam combines the established anti-pseudomonal cephalosporin, ceftazidime, with the novel non- $\beta$-lactam $\beta$-lactamase inhibitor, avibactam.

Objectives The aim of this study was to evaluate the safety of ceftazidime-avibactam in adults using pooled data from two phase II (NCT00690378, NCT00752219) and five phase III (NCT01499290, NCT01726023, NCT01644643, NCT01808093 and NCT01595438/NCT01599806) clinical studies.

Methods Safety data from seven multicentre, randomised, active-comparator studies were pooled by study group at the patient level for descriptive analyses, comprising patients with complicated urinary tract infection (cUTI), including pyelonephritis, complicated intra-abdominal infection (cIAI), or nosocomial pneumonia (NP), including ventilator-associated pneumonia (VAP), treated with ceftazidime-avibactam \pm metronidazole or comparator.

Results In total, 4050 patients (ceftazidime-avibactam \pm metronidazole, $n=2024$; comparator, $n=2026$ ) were included in the pooled analysis. Adverse events (AEs) up to the last study visit occurred in 996 (49.2\%) and 965 (47.6\%) patients treated with ceftazidime-avibactam \pm metronidazole and comparator, respectively. The most common AEs across treatment groups were diarrhoea, nausea, headache, vomiting and pyrexia. There were few discontinuations due to AEs $(2.5 \%$ and $1.7 \%$ for ceftazidime-avibactam \pm metronidazole and comparators, respectively). Overall rates of serious AEs were $8.7 \%$ for ceftazidime-avibactam \pm metronidazole and $7.2 \%$ for comparators; respective rates of AEs with an outcome of death were $2.0 \%$ and $1.8 \%$. AEs considered causally related to the study drug or procedures occurred in $10.7 \%$ and $9.6 \%$ of patients treated with ceftazidime-avibactam \pm metronidazole and comparators; the most common drug-related AEs in both groups were diarrhoea, headache, nausea and increased alanine aminotransferase. No impact to the safety profile of ceftazidimeavibactam \pm metronidazole was found with regard to intrinsic factors, such as age or renal function at baseline, or extrinsic factors, such as geographical origin. Potentially clinically significant changes in laboratory parameters were infrequent with no trends or safety concerns identified.

Conclusion The observed safety profile of ceftazidime-avibactam across infection types is consistent with the established safety profile of ceftazidime monotherapy and no new safety findings were identified. This analysis supports the use of ceftazidime-avibactam as a treatment option in adults with cUTI, cIAI and NP, including VAP.
\end{abstract}

Enhanced Digital Features To view enhanced digital features for this article go to: https://doi.org/10.6084/m9.figshare.12097713.

Electronic supplementary material The online version of this article (https://doi.org/10.1007/s40264-020-00934-3) contains supplementary material, which is available to authorized users.

Karen Cheng

karen.cheng@pfizer.com

Pfizer, Sandwich, Kent, UK

2 AstraZeneca, Alderley Park, Macclesfield, UK

3 Pfizer, Collegeville, PA, USA

\section{Key Points}

Safety and tolerability of ceftazidime-avibactam was similar to that of comparator drugs.

Safety data appeared to be consistent with that of historical data for ceftazidime alone.

Safety findings were similar between groups within different infections and post-marketing surveillance will continue to define safety in clinical practice. 


\section{Introduction}

With the increasing global prevalence of Gram-negative multidrug-resistant (MDR) bacteria, there is a need for the development of new antibiotics [1-4]. Ceftazidime is a third-generation cephalosporin with a well-established safety and tolerability profile. Like other $\beta$-lactam antibiotics, ceftazidime is susceptible to hydrolysis by $\beta$-lactamases that renders it inactive. The high and increasing prevalence of $\beta$-lactam resistance has reduced the utility of ceftazidime in many countries. Avibactam is a recently developed first-in-class non- $\beta$-lactam $\beta$-lactamase inhibitor of serine $\beta$-lactamases with a spectrum of inhibition that includes several mechanisms of resistance in bacteria. The addition of avibactam to ceftazidime expands its antibacterial activity against pathogens possessing $\beta$-lactamases that are susceptible to inhibition by avibactam, while retaining the bactericidal activity of ceftazidime [5]; it therefore has the potential to restore the utility of ceftazidime in the clinical setting. The focus of the safety assessment is to ascertain whether the addition of avibactam has an impact on the known, mature safety profile of ceftazidime monotherapy.

The efficacy and safety of ceftazidime-avibactam have been investigated in an adult clinical trial programme comprising 12 phase I [6-15], two phase II [16, 17] and five phase III studies [18-22]. The phase II and III programme included patients with serious life-threatening infections predominantly caused by Gram-negative bacteria; specifically, complicated intra-abdominal infection (cIAI), complicated urinary tract infection (cUTI), including acute pyelonephritis, and nosocomial pneumonia (NP), including ventilatorassociated pneumonia (VAP). The individual efficacy and safety results for each study have been presented [16-22]. Across the trials, ceftazidime-avibactam demonstrated a safety profile consistent with that of ceftazidime alone (known adverse drug reactions [ADRs] for ceftazidime intravenous administration are listed in Online Resource 1 [see Electronic Supplementary Material (ESM)]; those occurring with a frequency of $>1 \%$ include eosinophilia, thrombocytosis, phlebitis or thrombophlebitis, diarrhoea, transient increases in hepatic enzymes, maculopapular or urticarial rash and positive Coomb's test [23]).

This descriptive analysis of pooled safety data from patients enrolled in the seven phase II and III adult clinical studies was undertaken to characterise the overall safety and tolerability profile of ceftazidime-avibactam. It was structured in line with the Council for International Organizations of Medical Sciences (CIOMS) Working Group IV Management of Safety Information from Clinical Trials recommendations [24], and the individual trials were designed with standardisation of safety data collection with the intention of pooling the data to create the largest denominator set for each indication. It included sub-analyses of the impact of intrinsic and extrinsic patient factors on safety based on International Council for Harmonisation of Technical Requirements for Pharmaceuticals for Human Use (ICH) guidance [25], and assessments of pre-defined safety topics of special interest (STOI) based on the known safety profile of ceftazidime monotherapy. A recent meta-analysis of these published trials reported that serious adverse events (SAEs) were significantly more common for patients treated with ceftazidime-avibactam than for those in the pooled comparator group [26]; however, that analysis excluded 62 patients with moderate or severe renal impairment who were analysed separately in one of the phase III trials. The current analysis uses valid denominators (and a larger sample of patients with renal impairment) to provide more accurate treatment-emergent incidences for the 4050 patients who received at least one dose of study therapy in the adult phase II and III programme.

\section{Methods}

\subsection{Studies}

All studies included in the ceftazidime-avibactam adult phase II and III programme were multicentre, randomised, active-comparator trials (see Online Resource 2 in the ESM), which enrolled patients with cUTI, including pyelonephritis $[16,18,19]$, cIAI $[19,20,21]$ and NP, including VAP [22]. All studies were double-blind, except for the phase III REPRISE study in patients with cUTI or cIAI due to resistant pathogens, which was open-label [19], and the phase II NXL104-2001 study in patients with cUTI, which was investigator-blinded [16].

In the phase III studies, patients were randomised $(1: 1)$ to receive ceftazidime-avibactam (ceftazidime $2000 \mathrm{mg}$ and avibactam $500 \mathrm{mg}$ ) every $8 \mathrm{~h}$ (q8h) by 2-h intravenous (IV) infusions or comparator therapy for 5-21 days (duration of therapy was study-dependent, see Online Resource 2 in the ESM). In the phase II studies, the ceftazidime-avibactam dosage regimen was $500-125 \mathrm{mg} \mathrm{q} 8 \mathrm{~h}$ in the cUTI study and $2000-500 \mathrm{mg} \mathrm{q} 8 \mathrm{~h}$ in the cIAI study, with both studies using 30-min IV infusions. In the cIAI studies, patients randomised to ceftazidime-avibactam also received metronidazole (500 $\mathrm{mg}$ q8h by 60 -min IV infusions). In the phase III studies, the ceftazidime-avibactam dosage regimen was adjusted for patients with baseline creatinine clearance $(\mathrm{CrCl})<50 \mathrm{~mL} / \mathrm{min}$ and renal function was monitored during therapy (comparator dosage regimens, including adjustments for renal function, were based on approved product labelling). Patients with impaired renal function $(\mathrm{CrCl}<50 \mathrm{~mL} / \mathrm{min}$ in the cIAI study and $<70 \mathrm{~mL} / \mathrm{min}$ in the cUTI study) were excluded from the phase II studies [16, 
17]. For coverage of Gram-positive pathogens, linezolid, daptomycin or vancomycin could be co-administered with ceftazidime-avibactam. In addition, in the REPROVE trial, concurrent aminoglycoside treatment was given to approximately $80 \%$ of patients with suspected/confirmed MDR Gram-negative pathogens [22].

\subsection{Data}

Collection of safety data from the signing of the study consent to the final study visit was standardised across the programme with the intention to conduct pooled analyses in line with CIOMS IV recommendations [24] (for example, with respect to coding conventions, exclusion of events clearly identified to be associated with disease progression or asymptomatic abnormalities of protocolled laboratory parameters). Each study was reported using the MedDRA dictionary version for the time the respective study database was locked. For the present analysis, these were harmonised to a single dictionary version (MedDRA 19.0).

Safety variables included the incidence of adverse events (AEs) in patients taking ceftazidime-avibactam \pm metronidazole or comparator up to the last study visit categorised by MedDRA Preferred Term (PT), relationship to treatment of AEs (determined by study investigators who in all studies, apart from the open-label REPRISE study, were blinded to treatment), SAEs, discontinuations due to AEs, all-cause mortality and AEs with an outcome of death. Treatmentemergent AEs were defined as those occurring in any patient who received part of a dose of study therapy. AE intensity was determined by the investigators according to predefined criteria: mild (patient was aware of the symptoms but could tolerate them easily without a clinically significant effect on their health or requirement for medical intervention); moderate (AEs caused enough discomfort to interfere with usual activity or clinical status, possibly requiring medical intervention); or severe (AE was considered to be incapacitating or to have a significant effect on clinical status, with the likely need for medical intervention and close follow-up). SAEs were defined as any AE that was life-threatening or resulted in death, hospitalisation or prolongation of hospitalisation, persistent/significant disability or incapacity, congenital anomaly, birth defect or another important medical event that jeopardised the patient or required intervention.

Pre-planned sub-analyses of intrinsic and extrinsic factors were programmed for AE reporting at end of study therapy and up to last visit by gender, age group, race, baseline body mass index (BMI), baseline renal status, region, ethnicity and co-administration with drugs that potentially could result in a drug-drug interaction (DDI); within each of these sub-analyses, frequencies and severities of individual PTs across subgroups and indications were summarised.
Predefined safety topics of special interest (STOI) were also analysed, based on definitions created from Standardised MedDRA Queries comprising liver disorders, diarrhoea, hypersensitivity/anaphylaxis, haematological disorders representing low blood counts, and renal disorders. Laboratory parameters in the pooled dataset were analysed using shift from baseline and potentially clinically significant values based on pre-defined criteria. Known ADRs were defined using a combination of MedDRA PTs and laboratory definitions, and incidences were calculated.

\subsection{Statistics}

Safety data were pooled at the patient level across studies according to treatment group and summarised descriptively. The studies and the pooling were not designed to exclude bias associated with inferential testing of safety endpoints; therefore, no inferential statistical analyses were conducted.

\section{Results}

\subsection{Patients}

The pooled safety population included 4050 patients who received any amount of ceftazidime-avibactam or comparator. Of these, 2024 received ceftazidime-avibactam, including 857 patients with cIAI (who also received metronidazole), 731 patients with cUTI and 436 patients with NP; 2026 patients received comparator treatment, including 863 patients with cIAI, 729 patients with cUTI and 434 patients with NP. In nearly all cases, the comparator was a carbapenem (see Online Resource 2 in the ESM). An overview of patient disposition across the studies is shown in Fig. 1.

Across the cIAI, cUTI and NP cohorts (Table 1), the mean age was approximately 54 years, with $33 \%$ aged $\geq 65$ years. More than half of patients with NP/VAP were elderly ( $>65$ years), with $129 / 436$ $(29.6 \%) \geq 75$ years, whereas most patients with cIAI (389/857 [80.4\%]) were $<65$ years. In the cUTI cohort, ages were more evenly spread, with similar proportions aged $<45$ years, $46-64$ years and $\geq 65$ years. Most patients (approximately 62\%) were white in the cIAI and cUTI populations, with the largest proportion (approximately 49\%) from Eastern Europe, and most were Asian in the NP population, with 143 (32.8\%) from sites in mainland China. The mean BMI was approximately $25 \mathrm{~kg} /$ $\mathrm{m}^{2}$. Approximately half of patients overall had normal renal function (estimated $\mathrm{CrCl} \geq 81 \mathrm{~mL} / \mathrm{min}$ ) at baseline. Patients with mild renal impairment at baseline ( $\mathrm{CrCl} 51-80 \mathrm{~mL} / \mathrm{min}$ ) comprised approximately $27 \%$ of patients with cIAI and $38 \%$ of patients with cUTI and NP. 


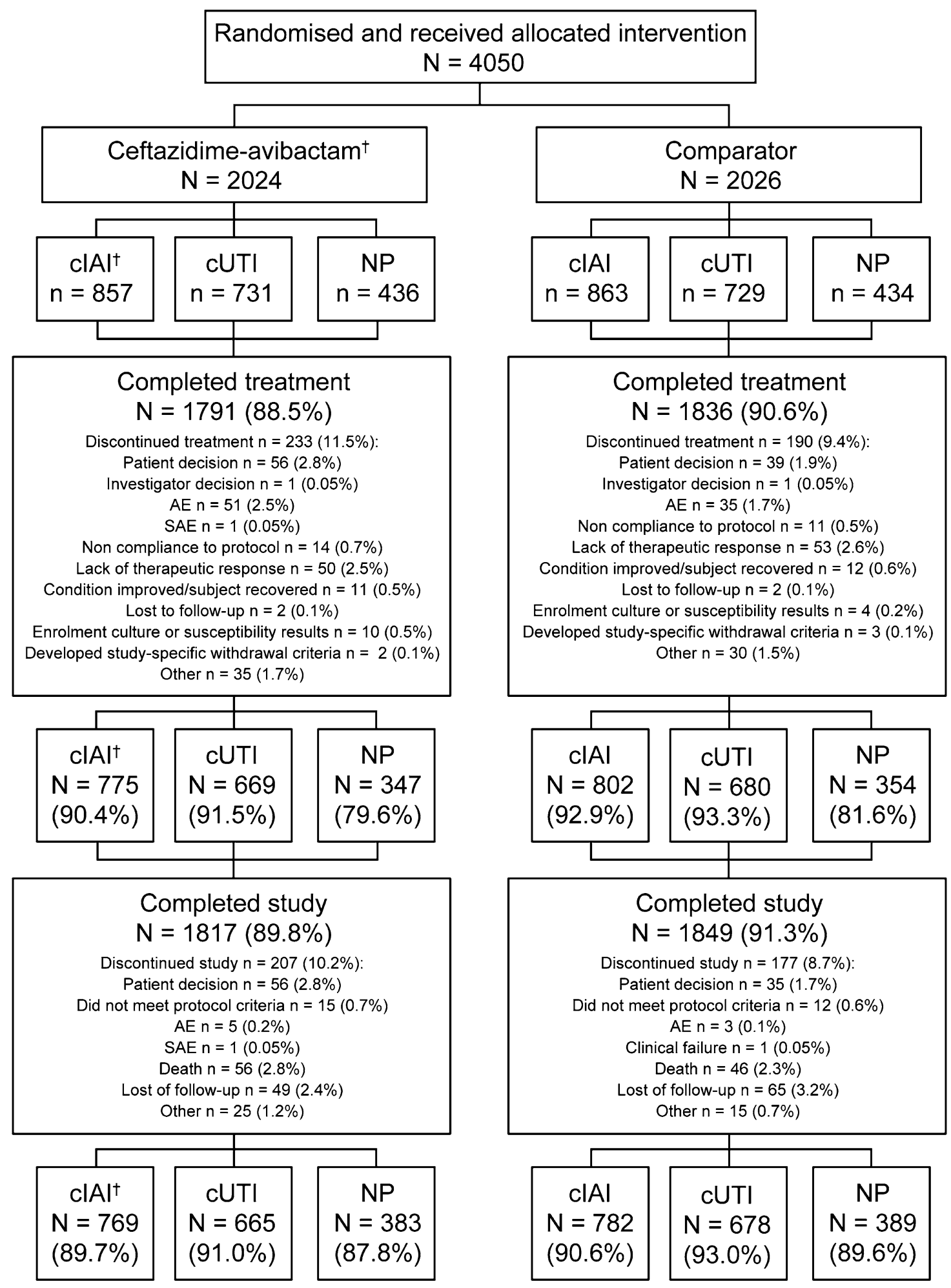

Fig. 1 Flow chart of patients included in the pooled phase II and III safety analysis (pooled safety population). $A E$ adverse event, cIAI complicated intra-abdominal infection, $c U T I$ complicated urinary

tract infection, $N P$ nosocomial pneumonia, $S A E$ serious adverse event. ${ }^{\dagger}$ Patients with cIAI received ceftazidime-avibactam in combination with metronidazole 
Patients with moderate-to-severe renal impairment (estimated $\mathrm{CrCl}<50 \mathrm{~mL} / \mathrm{min}$ ) at baseline were not recruited to all studies and comprised $6.7 \%$ of patients with cIAI and approximately $10 \%$ of the cUTI and NP/VAP populations (Table 1). Mean baseline Acute Physiology and Chronic Health Evaluation II (APACHE II) scores were higher in patients with NP/VAP than in patients with cIAI (cIAI: 6.6 vs 6.5 in RECLAIM, 4.8 vs 5.2 in RECLAIM 3 and 7.0 vs 9.4 in the REPRISE cIAI subgroup; NP: 14.5 vs 14.9 in REPROVE). APACHE II scores were not recorded in patients with cUTI.

\subsection{Duration of Treatment}

Overall, the mean \pm standard deviation (range) duration of treatment was $8.2 \pm 3.2(1-21)$ days in the ceftazidime-avibactam \pm metronidazole group and $8.4 \pm 3.2(1-23)$ days in the comparator group. Among patients with cIAI, cUTI and NP treated with ceftazidime-avibactam (plus metronidazole for cIAI patients), the mean (range) treatment durations were 7.7 (1-21), $7.9(1-21)$ and 9.5 (1-14) days, respectively, reflecting in part the clinical trials' protocol-specific differences in treatment duration (see Online Resource 2 in the ESM); corresponding values in the comparator groups were 8.0 (1-23), 8.1 (1-21) and 9.7 (1-15) days, respectively. The most common reasons for treatment discontinuation in the ceftazidime-avibactam \pm metronidazole and comparator groups, respectively, were subject decision (2.8\% and $1.9 \%)$, $\mathrm{AE}(2.5 \%$ and $1.7 \%)$ and lack of therapeutic response $(2.5 \%$ and 2.6\%). Overall, 1791/2024 patients (88.5\%) in the ceftazidime-avibactam \pm metronidazole group and 1836/2026 patients $(90.6 \%)$ in the comparator group completed treatment (Fig. 1).

\subsection{Adverse Events}

Overall, $49.2 \%$ of patients in the ceftazidime-avibactam \pm metronidazole group and $47.6 \%$ of those in the comparator group had any AE up to the last study visit (Table 2), and most AEs were of mild or moderate intensity and did not result in discontinuation of study therapy. The incidence of patients with any AE up to the last study visit was balanced across treatment groups, with patients with cUTI having the lowest incidence and patients with NP/VAP having the highest incidence (Table 2). The incidences of AEs occurring up to the end of study therapy (EOT) were similar to those occurring up to the last visit (872/2024 [43.1\%] for ceftazidime-avibactam \pm metronidazole and 847/2026 [41.8\%] for comparator).

The most common AEs overall and within each indication in the ceftazidime-avibactam \pm metronidazole group were diarrhoea, nausea, and headache (Table 3). In patients with
NP/VAP, there was increased reporting of hypokalaemia as an AE compared with those with cUTI and cIAI; however, laboratory analysis of actual shifts in serum potassium during treatment did not indicate a treatment effect (see Online Resource 3 in ESM).

In the ceftazidime-avibactam \pm metronidazole group, the incidence of AEs of infusion-site phlebitis up to the last study visit (defined using the PTs Administration site erythema, Administration site inflammation, Administration site phlebitis, Infusion site inflammation, Infusion site pain, Infusion site phlebitis, Injection site erythema, Injection site phlebitis, Vessel puncture site erythema, and Vessel puncture site phlebitis) was $0.4 \%$ and the incidence of AEs of infusion site thrombosis (defined using the PTs Administration site bruise, Administration site haematoma, Administration site thrombosis, Infusion site bruising, Infusion site haematoma, Infusion site thrombosis, Injection site bruising, Injection site haematoma, Injection site thrombosis) was $0.05 \%$. In addition, the PTs Injection site pain, Infusion site erythema, Thrombophlebitis, Thrombophlebitis superficial, Phlebitis, and Phlebitis superficial up to EOT each occurred in $<1 \%$ of patients; however, there was insufficient information to confirm if these AEs were related to the infusion site or thrombophlebitis, all were non-serious, and most were of mild intensity. Similar rates of infusion-site phlebitis and infusion-site thrombosis PTs were seen in the comparator groups (data not shown).

The incidence of AEs assessed by the investigator to be related to the study drug or procedures was $217 / 2014$ $(10.7 \%)$ for ceftazidime-avibactam \pm metronidazole and $194 / 2026$ (9.6\%) for the comparator group. The most common ( $\geq 1 \%$ in either treatment group) treatment-related AEs in both groups were diarrhoea, headache, nausea and increased alanine aminotransferase (ALT) (see Online Resource 4 in the ESM), all of which are known ADRs of ceftazidime [23]. Analysis of the AEs showed a pattern consistent with the known safety profile of ceftazidime, the underlying infection or the patient population.

Overall, SAEs occurred in 176/2024 (8.7\%) and 145/2026 $(7.2 \%)$ patients in the ceftazidime-avibactam \pm metronidazole and comparator groups, respectively, and, like that of overall AEs, the lowest and highest incidences of SAEs were reported by patients with cUTI and NP, respectively (Table 2). The most common SAEs up to the last visit (ceftazidime-avibactam \pm metronidazole group) were pneumonia $(0.5 \%)$, acute kidney injury $(0.4 \%)$, respiratory failure $(0.4 \%)$ and sepsis $(0.3 \%)$. Few SAEs were assessed by the investigator to be related to the study drug, and treatmentrelated SAEs were sporadic in type (Table 4).

Overall, 64/2024 (3.2\%) patients in the ceftazidime-avibactam \pm metronidazole group and 53/2026 (2.6\%) patients in the comparator group died up to the last scheduled follow-up visit. Of these deaths, 30 occurred in 
Table 1 Baseline demographics and disease characteristics (pooled safety population)

\begin{tabular}{|c|c|c|c|c|c|c|c|c|}
\hline \multirow[t]{2}{*}{ Characteristic } & \multicolumn{2}{|l|}{ cIAI } & \multicolumn{2}{|l|}{ cUTI } & \multicolumn{2}{|c|}{ NP including VAP } & \multicolumn{2}{|l|}{ Overall } \\
\hline & $\begin{array}{l}\text { Ceftazidime- } \\
\text { avibactam }+ \\
\text { metronida- } \\
\text { zole }(N=857)\end{array}$ & $\begin{array}{l}\text { Comparator } \\
(N=863)\end{array}$ & $\begin{array}{l}\text { Ceftazidime- } \\
\text { avibactam } \\
(N=731)\end{array}$ & $\begin{array}{l}\text { Comparator } \\
(N=729)\end{array}$ & $\begin{array}{l}\text { Ceftazidime- } \\
\text { avibactam } \\
(N=436)\end{array}$ & $\begin{array}{l}\text { Meropenem } \\
(N=434)\end{array}$ & $\begin{array}{l}\text { Ceftazidime- } \\
\text { avibactam } \pm \\
\text { metronidazole } \\
(N=2024)\end{array}$ & $\begin{array}{l}\text { Comparator } \\
(N=2026)\end{array}$ \\
\hline $\begin{array}{l}\text { Mean age }(\mathrm{SD}) \text {, } \\
\text { years }\end{array}$ & $48.6(17.2)$ & $49.0(18.2)$ & $53.8(19.5)$ & $53.9(18.5)$ & $62.8(16.7)$ & $62.8(17.6)$ & $53.5(18.7)$ & $53.7(18.9)$ \\
\hline \multicolumn{9}{|l|}{ Age group, $n(\%)$} \\
\hline$\geq 18-45$ years & $379(44.2)$ & $382(44.3)$ & $243(33.2)$ & $224(30.7)$ & $75(17.2)$ & $74(17.1)$ & $697(34.4)$ & $680(33.6)$ \\
\hline 46-64 years & $310(36.2)$ & $287(33.3)$ & $231(31.6)$ & $259(35.5)$ & $125(28.7)$ & $127(29.3)$ & $666(32.9)$ & $673(33.2)$ \\
\hline $65-74$ years & $107(12.5)$ & $110(12.7)$ & $133(18.2)$ & $146(20.0)$ & $107(24.5)$ & $98(22.6)$ & $347(17.1)$ & $354(17.5)$ \\
\hline$\geq 75$ to $\leq 90$ years & $61(7.1)$ & $84(9.7)$ & $124(17.0)$ & $100(13.7)$ & $129(29.6)$ & $135(31.1)$ & $314(15.5)$ & $319(15.7)$ \\
\hline Male, $n(\%)$ & $544(63.5)$ & 577 (66.9) & $264(36.1)$ & $250(34.3)$ & $325(74.5)$ & $320(73.7)$ & $1133(56.0)$ & $1147(56.6)$ \\
\hline \multicolumn{9}{|l|}{ Race, $n(\%)$} \\
\hline White & $472(55.1)$ & $477(55.3)$ & $602(82.4)$ & $611(83.8)$ & $181(41.5)$ & $189(43.5)$ & $1255(62.0)$ & $1277(63.0)$ \\
\hline $\begin{array}{l}\text { Black or African } \\
\text { American }\end{array}$ & $7(0.8)$ & $3(0.3)$ & $5(0.7)$ & $14(1.9)$ & $3(0.7)$ & $2(0.5)$ & $15(0.7)$ & $19(0.9)$ \\
\hline Asian & 331 (38.6) & 334 (38.7) & $60(8.2)$ & $43(5.9)$ & $245(56.2)$ & $236(54.4)$ & $636(31.4)$ & $613(30.3)$ \\
\hline $\begin{array}{l}\text { Native Hawaiian } \\
\text { or other Pacific } \\
\text { Islander }\end{array}$ & $0(0.0)$ & $0(0.0)$ & $0(0.0)$ & $0(0.0)$ & $1(0.2)$ & $0(0.0)$ & $1(0.0)$ & $0(0.0)$ \\
\hline $\begin{array}{l}\text { American Indian } \\
\text { or Alaskan } \\
\text { Native }\end{array}$ & $6(0.7)$ & $6(0.7)$ & $1(0.1)$ & $3(0.4)$ & $0(0.0)$ & $0(0.0)$ & $7(0.3)$ & $9(0.4)$ \\
\hline Other & $39(4.6)$ & $43(5.0)$ & $63(8.6)$ & $58(8.0)$ & $6(1.4)$ & $7(1.6)$ & $108(5.3)$ & $108(5.3)$ \\
\hline Missing & $2(0.2)$ & $0(0.0)$ & $0(0.0)$ & $0(0.0)$ & $0(0.0)$ & $0(0.0)$ & $2(0.1)$ & $0(0.0)$ \\
\hline \multicolumn{9}{|l|}{ Region, $n(\%)$} \\
\hline Western Europe $^{\mathrm{a}}$ & $47(5.5)$ & $47(5.4)$ & $23(3.1)$ & $22(3.0)$ & $37(8.5)$ & $34(7.8)$ & $107(5.3)$ & $103(5.1)$ \\
\hline Eastern Europe ${ }^{\mathrm{b}}$ & $369(43.1)$ & $376(43.6)$ & $507(69.4)$ & $511(70.1)$ & $113(25.9)$ & $109(25.1)$ & $989(48.9)$ & $996(49.2)$ \\
\hline China & $130(15.2)$ & $136(15.8)$ & $0(0.0)$ & $0(0.0)$ & $143(32.8)$ & $145(33.4)$ & $273(13.5)$ & $281(13.9)$ \\
\hline Rest of World ${ }^{\mathrm{c}}$ & $311(36.3)$ & 304 (35.2) & $201(27.5)$ & $196(26.9)$ & $143(32.8)$ & $146(33.6)$ & $655(32.4)$ & $646(31.9)$ \\
\hline $\begin{array}{l}\text { Mean body mass } \\
\text { index (SD), } \mathrm{kg} / \mathrm{m}^{2}\end{array}$ & $25.09(5.0)^{\mathrm{d}}$ & $25.14(4.9)^{\mathrm{e}}$ & $26.69(6.0)^{\mathrm{f}}$ & $26.72(5.8)^{\mathrm{g}}$ & $23.78(6.0)^{\mathrm{h}}$ & $23.60(5.2)^{\mathrm{i}}$ & $25.39(5.7)^{\mathrm{j}}$ & $25.38(5.4)^{\mathrm{k}}$ \\
\hline \multicolumn{9}{|c|}{$\mathrm{CrCl}$ group $(\mathrm{mL} / \mathrm{min}), n(\%)$} \\
\hline$\geq 81$ & $561(65.5)$ & $576(66.7)$ & $362(49.5)$ & 367 (50.3) & 213 (48.9) & $214(49.3)$ & $1136(56.1)$ & $1157(57.1)$ \\
\hline $51-80$ & $234(27.3)$ & $212(24.6)$ & $281(38.4)$ & $280(38.4)$ & $166(38.1)$ & $164(37.8)$ & $681(33.6)$ & $656(32.4)$ \\
\hline $31-50$ & $55(6.4)$ & $70(8.1)$ & $77(10.5)$ & $74(10.2)$ & 43 (9.9) & $46(10.6)$ & $175(8.6)$ & $190(9.4)$ \\
\hline$\leq 30$ & $2(0.2)$ & $3(0.3)$ & $8(1.1)$ & $7(1.0)$ & $12(2.8)$ & $8(1.8)$ & $22(1.1)$ & $18(0.9)$ \\
\hline Missing & $5(0.6)$ & $2(0.2)$ & $3(0.4)$ & $1(0.1)$ & $2(0.5)$ & $2(0.5)$ & $10(0.5)$ & $5(0.2)$ \\
\hline
\end{tabular}

cIAI complicated intra-abdominal infection, $\mathrm{CrCl}$ creatinine clearance, $c U T I$ complicated urinary tract infection, $n$ number of patients, $N$ number of patients in treatment group, $N P$ nosocomial pneumonia, $S D$ standard deviation, $V A P$ ventilator-associated pneumonia

${ }^{a}$ Includes Belgium, France, Germany, Greece, Italy, the Netherlands, Portugal, Spain and the United Kingdom

${ }^{b}$ Includes Bulgaria, Croatia, Czech Republic, Hungary, Latvia, Lithuania, Poland, Romania, Russian Federation, Serbia, Slovakia, Turkey and Ukraine

${ }^{\mathrm{c}}$ Includes Argentina, Brazil, Canada, Chile, India, Israel, Lebanon, Mexico, Malaysia, Peru, Thailand, Taiwan, the United States, Guatemala, Jordan, South Korea, Japan, Vietnam, the Philippines and South Africa

${ }^{\mathrm{d}} n=849$

$\mathrm{e}_{n=855}$

${ }^{\mathrm{f}} n=723$

$\mathrm{g}_{n=723}$

$\mathrm{h}_{n}=425$

${ }^{\mathrm{i}} n=423$

$\mathrm{j}_{n=1997}$

${ }^{\mathrm{k}} n=2001$ 
Table 2 Adverse events up to the last study visit (pooled safety population)

\begin{tabular}{|c|c|c|c|c|c|c|c|c|}
\hline \multirow{2}{*}{$\begin{array}{l}\text { AE category, } \\
n(\%)\end{array}$} & \multicolumn{2}{|l|}{ cIAI } & \multicolumn{2}{|l|}{ cUTI } & \multicolumn{2}{|c|}{ NP including VAP } & \multicolumn{2}{|l|}{ Total } \\
\hline & $\begin{array}{l}\text { Ceftazidime- } \\
\text { avibactam }+ \\
\text { metronidazole } \\
(N=857)\end{array}$ & $\begin{array}{l}\text { Comparator } \\
(N=863)\end{array}$ & $\begin{array}{l}\text { Ceftazidime- } \\
\text { avibactam } \\
(N=731)\end{array}$ & $\begin{array}{l}\text { Comparator } \\
(N=729)\end{array}$ & $\begin{array}{l}\text { Ceftazidime- } \\
\text { avibactam } \\
(N=436)\end{array}$ & $\begin{array}{l}\text { Meropenem } \\
(N=434)\end{array}$ & $\begin{array}{l}\text { Ceftazidime- } \\
\text { avibactam } \pm \\
\text { metronidazole } \\
(N=2024)\end{array}$ & $\begin{array}{l}\text { Comparator } \\
(N=2026)\end{array}$ \\
\hline Any AE & $398(46.4)$ & $381(44.1)$ & 275 (37.6) & $263(36.1)$ & $323(74.1)$ & $321(74.0)$ & $996(49.2)$ & 965 (47.6) \\
\hline $\begin{array}{l}\text { Any AE with } \\
\text { outcome of } \\
\text { death }^{\mathrm{a}}\end{array}$ & $10(1.2)$ & $9(1.0)$ & $3(0.4)$ & $4(0.5)$ & $27(6.2)$ & $24(5.5)$ & $40(2.0)$ & $37(1.8)$ \\
\hline Any SAE & $62(7.2)$ & $67(7.8)$ & $35(4.8)$ & $19(2.6)$ & $79(18.1)$ & 59 (13.6) & $176(8.7)$ & $145(7.2)$ \\
\hline $\begin{array}{l}\text { Discontinua- } \\
\text { tion of study } \\
\text { drug due to } \\
\text { AEs }\end{array}$ & $26(3.0)$ & $14(1.6)$ & $10(1.4)$ & $7(1.0)$ & $16(3.7)$ & $13(3.0)$ & $52(2.6)$ & 34 (1.7) \\
\hline $\begin{array}{l}\text { AEs of severe } \\
\text { intensity }\end{array}$ & $41(4.8)$ & $54(6.3)$ & $16(2.2)$ & $18(2.5)$ & $68(15.6)$ & $55(12.7)$ & $125(6.2)$ & $127(6.3)$ \\
\hline
\end{tabular}

Patients with multiple AEs in the same category were counted once in that category; those with AEs in more than one category were counted in each category

$A E$ adverse event, $c I A I$ complicated intra-abdominal infection, $c U T I$ complicated urinary tract infection, $n$ number of patients, $N$ number of patients in treatment group, NP nosocomial pneumonia, SAE serious adverse event, VAP ventilator-associated pneumonia

${ }^{a}$ Excluding deaths due to disease progression. Two additional patients, both with a diagnosis of cIAI, died after the last scheduled follow-up visit. One of these patients was a 66-year-old female with a history of diabetes mellitus. This patient received ceftazidime-avibactam plus metronidazole and had an SAE of myocardial infarction on day 39, which resulted in death on day 61. The second patient was a 54-year-old male with a sigmoid perforation and septic shock. This patient received ceftazidime-avibactam plus metronidazole. On day 67 , he suffered a sudden respiratory and cardiac arrest that resulted in death

patients with cIAI (18 [2.1\%] in the ceftazidime-avibactam \pm metronidazole group and $12[1.4 \%]$ in the comparator group), seven in patients with cUTI (three [0.4\%] and four [0.5\%], respectively) and 80 in patients with NP/VAP (43 [9.9\%] and 37 [8.5\%], respectively). Forty patients $(2.0 \%)$ in the ceftazidime-avibactam \pm metronidazole group and $37(1.8 \%)$ in the comparator group experienced AEs with an outcome of death; none of these AEs were considered by the investigator to be related to the study drug. The remaining deaths were due to disease progression and occurred in $24(1.2 \%)$ and $16(0.8 \%)$ patients in the ceftazidime-avibactam \pm metronidazole and comparator groups, respectively (eight and three in patients with cIAI; none in patients with cUTI; 16 and 13, respectively, in patients with NP/VAP). Two additional patients, both with cIAI, died after the last scheduled follow-up visit (see the footnote to Table 2 for additional information). These were both considered by the blinded investigator to be unrelated to study medication and were attributed to the patients' pre-existing conditions.

The incidence of study drug discontinuation due to an $\mathrm{AE}$ was 2.6\% (52/2024) and 1.7\% (34/2026) in the ceftazidime-avibactam \pm metronidazole and comparator groups, respectively (Table 2). The most frequently reported AEs leading to discontinuation of ceftazidime-avibactam were nausea $(n=4)$, diarrhoea $(n=3)$ and headache $(n=2)$.

\subsection{Safety Topics of Special Interest}

STOI were defined a priori for close analysis in the clinical development programme, based on the known safety profile of ceftazidime. These were liver disorders, diarrhoea, severe hypersensitivity, haematological disorders and renal disorders (including neurological sequalae). Few patients in either group had STOI AEs (see Online Resource 5 in the ESM).

\subsubsection{Liver disorders}

Liver disorders were defined as an STOI based on the recognised ADRs for ceftazidime, which include transient elevations in one or more hepatic enzymes and jaundice [23]. The most common liver disorder AEs in the ceftazidime-avibac$\operatorname{tam} \pm$ metronidazole and comparator groups were increased aspartate transaminase (AST) $(1.8 \%$ and $2.0 \%$, respectively) and increased ALT (1.7\% and 2.1\%, respectively; Table 3). Patients with NP/VAP had the highest incidence of liver 


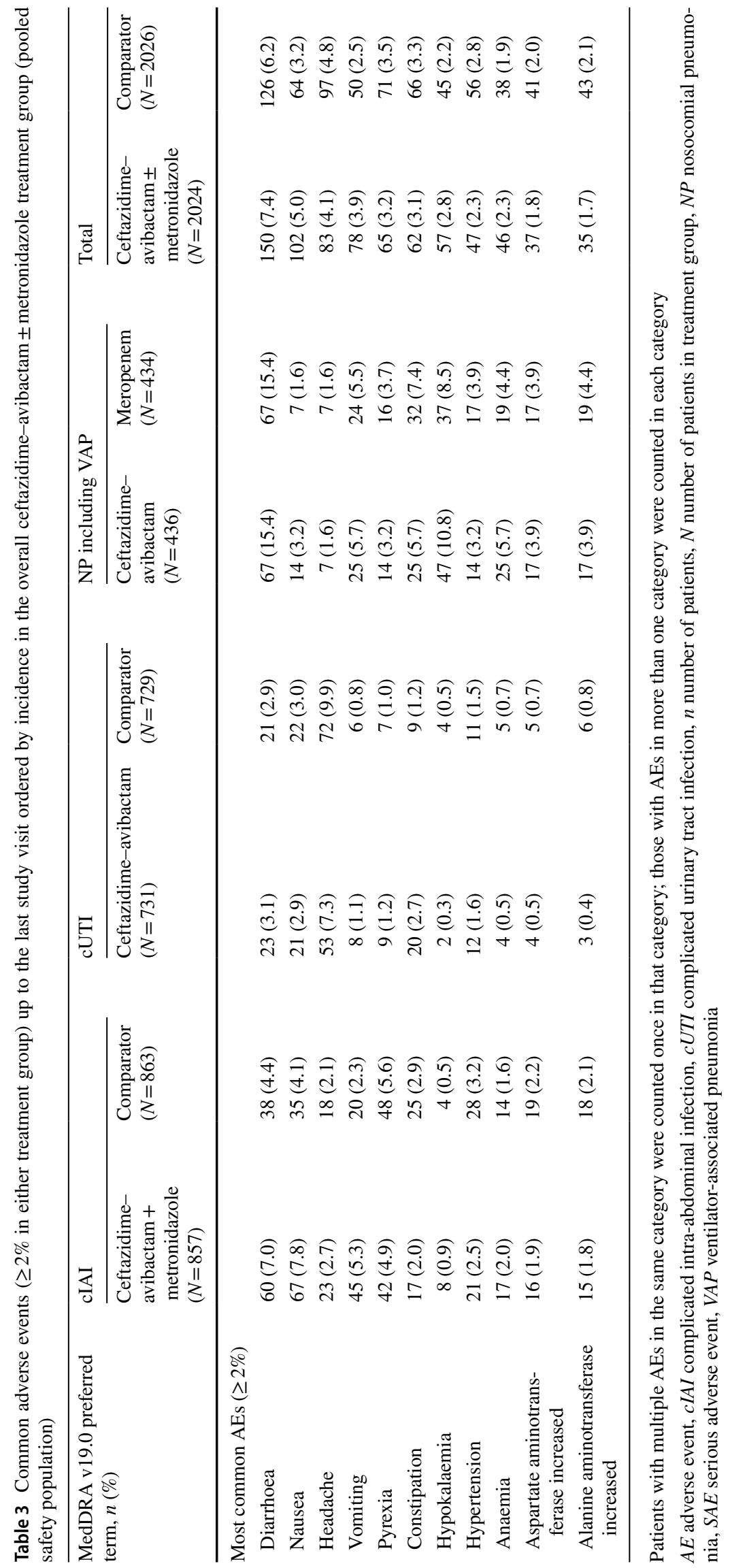


Table 4 Serious adverse events up to the last study visit considered by the blinded investigators to be related to the study drug ordered by incidence in the overall ceftazidimeavibactam \pm metronidazole treatment group (pooled safety population)

\begin{tabular}{|c|c|c|}
\hline MedDRA v19.0 preferred term, $n(\%)$ & $\begin{array}{l}\text { Ceftazidime-avibactam } \pm \\
\text { metronidazole }(N=2024)\end{array}$ & $\begin{array}{l}\text { Comparator } \\
(N=2026)\end{array}$ \\
\hline Diarrhoea $^{\mathrm{a}, \mathrm{b}}$ & $2(0.1)$ & $0(0.0)$ \\
\hline Accidental overdose & $1(0.0)$ & $0(0.0)$ \\
\hline Acute coronary syndrome & $1(0.0)$ & $0(0.0)$ \\
\hline Acute kidney injury ${ }^{\mathrm{a}}$ & $1(0.0)$ & $0(0.0)$ \\
\hline Alanine aminotransferase increased $\mathrm{d}^{\mathrm{a}, \mathrm{b}}$ & $1(0.0)$ & $0(0.0)$ \\
\hline Aspartate aminotransferase increased $\mathrm{d}^{\mathrm{a}, \mathrm{b}}$ & $1(0.0)$ & $0(0.0)$ \\
\hline Chronic hepatitis $\mathrm{C}$ & $1(0.0)$ & $0(0.0)$ \\
\hline Hepatic enzyme increased ${ }^{\mathrm{a}, \mathrm{b}}$ & $1(0.0)$ & $0(0.0)$ \\
\hline Hepatic function abnormal ${ }^{\mathrm{a}, \mathrm{b}}$ & $1(0.0)$ & $0(0.0)$ \\
\hline Hypersensitivity $^{\mathrm{a}}$ & $1(0.0)$ & $0(0.0)$ \\
\hline Liver function test abnormal ${ }^{a, b}$ & $1(0.0)$ & $0(0.0)$ \\
\hline Pyrexia ${ }^{a, b}$ & $1(0.0)$ & $0(0.0)$ \\
\hline Subacute hepatic failure & $1(0.0)$ & $0(0.0)$ \\
\hline Blood creatinine increased ${ }^{a}$ & $0(0.0)$ & $1(0.0)$ \\
\hline Clostridium difficile colitis ${ }^{\mathrm{a}}$ & $0(0.0)$ & $1(0.0)$ \\
\hline Confusional state $^{\mathrm{a}}$ & $0(0.0)$ & $1(0.0)$ \\
\hline Drug eruption & $0(0.0)$ & $1(0.0)$ \\
\hline Hyperkalaemia & $0(0.0)$ & $1(0.0)$ \\
\hline Transaminases increased ${ }^{\mathrm{a}, \mathrm{b}}$ & $0(0.0)$ & $1(0.0)$ \\
\hline
\end{tabular}

The investigator was blinded in all studies except for the open-label REPRISE study

$n$ number of patients, $N$ number of patients in treatment group

${ }^{a}$ Adverse events that are considered to be listed for ceftazidime as defined in the Ceftazidime Summary of Product Characteristics 2016 [23]

${ }^{\mathrm{b}}$ Adverse events that are considered to be listed for metronidazole as defined in the Metronidazole Summary of Product Characteristics 2017 [31] disorder AEs. Overall, liver disorder AEs and/or abnormal hepatic laboratory results that were considered potentially clinically significant occurred in $<5 \%$ of patients in either treatment group (Table 5). No notable trends were observed by visit for any hepatic laboratory parameter, and the elevations were in line with the expected frequency for this ADR. Fifty-seven patients (27/2024 [1.3\%] ceftazidime-avibactam \pm metronidazole and 30/2026 [1.5\%] comparator) met the laboratory criteria for potential Hy's law classification $(\geq 1$ laboratory value with a maximum ALT or AST $\geq 3 \times$ upper limit of normal [ULN] and a maximum total bilirubin level $\geq 2 \times U L N$ at any point, including before the start of study drug administration, up to the last visit). Assessment of these cases concluded that all patients had other aetiologies or explanations for the elevated liver results. No Hy's law cases were confirmed. No new safety findings were identified for liver disorders. Drug-induced liver injury was not confirmed in patients taking ceftazidime-avibactam \pm metronidazole. Hepatoxicity is considered an important potential risk for ceftazidime-avibactam in the Patient Risk Management Plan and is under pharmacovigilance surveillance [27].

\subsubsection{Diarrhoea}

Antibiotics are known to cause effects on the gastrointestinal tract that may result in alterations of intestinal flora and lead to antibiotic-associated diarrhoea, including Clostridium difficile colitis. According to the protocols of some of the phase III studies, if a patient experienced significant diarrhoea during or after IV study therapy, the investigator was to consider obtaining a stool sample for $C$. difficile toxin testing. In total, 154 patients $(7.6 \%)$ in the ceftazidime-avibactam \pm metronidazole and 132 patients $(6.5 \%)$ in the comparator group experienced diarrhoea AEs (see Online Resource 5 in the ESM); in the cIAI population, diarrhoea AEs occurred in 62 (7.2\%) and 38 (4.4\%) patients, respectively. Overall, there were 14 AEs in 13 patients potentially representing $C$. diffcile-associated diarrhoea (CDAD), nine occurring in eight patients who received ceftazidime-avibactam \pm metronidazole (one patient with cIAI, two with cUTI, and five with NP/VAP) and five occurring in five patients who received comparator (one patient each with cIAI and cUTI, and three with NP). All of these AEs had either resolved or were resolving at the time of reporting. 
Table 5 Potentially clinically significant laboratory measurements up to the last study visit (pooled safety population)

\begin{tabular}{|c|c|c|c|}
\hline \multirow[t]{2}{*}{ Clinical laboratory variable } & \multirow[t]{2}{*}{ Potentially clinically significant criteria } & \multicolumn{2}{|c|}{$\begin{array}{l}n / m \text { with both baseline and post- } \\
\text { baseline values }(\%)\end{array}$} \\
\hline & & $\begin{array}{l}\text { Ceftazidime- } \\
\text { avibactam } \pm \\
\text { metronidazole } \\
(N=2024)\end{array}$ & $\begin{array}{l}\text { Comparator } \\
(N=2026)\end{array}$ \\
\hline \multicolumn{4}{|l|}{ Haematology } \\
\hline Basophils, particle concentration $\left(10^{9} / \mathrm{L}\right)$ & $>4 \times$ ULN and $>300 \%$ increase from baseline & $1 / 1729(0.1)$ & $2 / 1732(0.1)$ \\
\hline Coombs test, direct & Negative at baseline and positive at post-baseline & $128 / 986(13.0)$ & $37 / 978(3.8)$ \\
\hline Eosinophils, particle concentration $\left(10^{9} / \mathrm{L}\right)$ & $>4 \times$ ULN and $>300 \%$ increase from baseline & $2 / 1736(0.1)$ & $3 / 1742(0.2)$ \\
\hline \multirow[t]{2}{*}{ Erythrocyte, volume fraction (ratio) } & $<0.8 \times$ LLN and $>20 \%$ decrease from baseline & $87 / 1578(5.5)$ & $94 / 1573(6.0)$ \\
\hline & $>1.3 \times \mathrm{ULN}$ and $>30 \%$ increase from baseline & $0 / 1578(0.0)$ & $0 / 1573(0.0)$ \\
\hline \multirow[t]{2}{*}{ Erythrocytes, particle concentration $\left(10^{12} / \mathrm{L}\right)$} & $<0.8 \times$ LLN and $>20 \%$ decrease from baseline & $95 / 1759(5.4)$ & $92 / 1772(5.2)$ \\
\hline & $>1.3 \times \mathrm{ULN}$ and $>30 \%$ increase from baseline & $1 / 1759(0.1)$ & $0 / 1772(0.0)$ \\
\hline \multirow[t]{2}{*}{ Haemoglobin $(\mathrm{g} / \mathrm{L})$} & $<0.8 \times$ LLN and $>20 \%$ decrease from baseline & $101 / 1760(5.7)$ & $100 / 1772(5.6)$ \\
\hline & $>1.3 \times$ ULN and $>30 \%$ increase from baseline & $0 / 1760(0.0)$ & $0 / 1772(0.0)$ \\
\hline \multirow[t]{2}{*}{ Leukocytes, particle concentration $\left(10^{9} / \mathrm{L}\right)$} & $<0.65 \times \mathrm{LLN}$ and $>60 \%$ decrease from baseline & $13 / 1759(0.7)$ & $13 / 1772(0.7)$ \\
\hline & $>1.6 \times \mathrm{ULN}$ and $>100 \%$ increase from baseline & $32 / 1759(1.8)$ & $37 / 1772(2.1)$ \\
\hline \multirow[t]{2}{*}{ Lymphocytes, particle concentration $\left(10^{9} / \mathrm{L}\right)$} & $<0.25 \times \mathrm{LLN}$ and $>75 \%$ decrease from baseline & $3 / 1747(0.2)$ & $6 / 1754(0.3)$ \\
\hline & $>1.5 \times \mathrm{ULN}$ and $>100 \%$ increase from baseline & $6 / 1747(0.3)$ & $11 / 1754(0.6)$ \\
\hline Monocytes, particle concentration $\left(10^{9} / \mathrm{L}\right)$ & $>4 \times$ ULN and $>300 \%$ increase from baseline & $1 / 1743(0.1)$ & $1 / 1746(0.1)$ \\
\hline \multirow[t]{2}{*}{ Neutrophils, particle concentration $\left(10^{9} / \mathrm{L}\right)$} & $<0.65 \times \mathrm{LLN}$ and $>75 \%$ decrease from baseline & $16 / 1743(0.9)$ & $17 / 1747(1.0)$ \\
\hline & $>1.6 \times \mathrm{ULN}$ and $>100 \%$ increase from baseline & $70 / 1743(4.0)$ & $59 / 1747(3.4)$ \\
\hline \multirow[t]{2}{*}{ Platelets, particle concentration $\left(10^{9} / \mathrm{L}\right)$} & $<0.65 \times \mathrm{LLN}$ and $>50 \%$ decrease from baseline & $17 / 1663(1.0)$ & $20 / 1673(1.2)$ \\
\hline & $>1.5 \times \mathrm{ULN}$ and $>100 \%$ increase from baseline & $59 / 1663(3.5)$ & $63 / 1673(3.8)$ \\
\hline \multicolumn{4}{|l|}{ Clinical chemistry } \\
\hline $\operatorname{ALT}(\mu \mathrm{kat} / \mathrm{L})$ & $>3 \times$ ULN and $>200 \%$ increase from baseline & $66 / 1818(3.6)$ & $71 / 1821(3.9)$ \\
\hline \multirow[t]{2}{*}{ Albumin $(\mathrm{g} / \mathrm{L})$} & $<0.5 \times$ LLN and $>50 \%$ decrease from baseline & 2/1837 (0.1) & $3 / 1829(0.2)$ \\
\hline & $>1.5 \times \mathrm{ULN}$ and $>50 \%$ increase from baseline & $0 / 1837(0.0)$ & $0 / 1829(0.0)$ \\
\hline \multirow[t]{2}{*}{$\operatorname{ALP}(\mu \mathrm{kat} / \mathrm{L})$} & $<0.5 \times$ LLN and $>80 \%$ decrease from baseline & $0 / 1919(0.0)$ & $0 / 1923(0.0)$ \\
\hline & $>2 \times$ ULN and $>100 \%$ increase from baseline & $51 / 1919(2.7)$ & $44 / 1923(2.3)$ \\
\hline AST $(\mu \mathrm{kat} / \mathrm{L})$ & $>3 \times$ ULN and $>200 \%$ increase from baseline & $63 / 1761(3.6)$ & $53 / 1757(3.0)$ \\
\hline Bilirubin, direct (conjugated) $(\mu \mathrm{mol} / \mathrm{L})$ & $>2.5 \times \mathrm{ULN}$ and $>150 \%$ increase from baseline & 4/1403 (0.3) & $17 / 1381(1.2)$ \\
\hline Total bilirubin $(\mu \mathrm{mol} / \mathrm{L})$ & $>2 \times \mathrm{ULN}$ and $>150 \%$ increase from baseline & $4 / 1841(0.2)$ & $13 / 1836(0.7)$ \\
\hline Creatinine $(\mu \mathrm{mol} / \mathrm{L})$ & $>2 \times \mathrm{ULN}$ and $>100 \%$ increase from baseline & $8 / 1929(0.4)$ & $12 / 1928(0.6)$ \\
\hline Gamma-glutamyltransferase ( $\mu \mathrm{kat} / \mathrm{L})$ & $>3 \times$ ULN and $>200 \%$ increase from baseline & $78 / 1763(4.4)$ & $84 / 1759(4.8)$ \\
\hline \multirow[t]{2}{*}{ Urea nitrogen $(\mathrm{mmol} / \mathrm{L})$} & $<0.2 \times \mathrm{LLN}$ and $>100 \%$ decrease from baseline & 0/1930 (0.0) & 0/1927 (0.0) \\
\hline & $>3 \times \mathrm{ULN}$ and $>200 \%$ increase from baseline & $3 / 1930(0.2)$ & $3 / 1927(0.2)$ \\
\hline \multirow[t]{2}{*}{ Potassium (mmol/L) } & $<0.8 \times$ LLN and $>20 \%$ decrease from baseline & $43 / 1893(2.3)$ & $39 / 1882(2.1)$ \\
\hline & $>1.2 \times \mathrm{ULN}$ and $>20 \%$ increase from baseline & $15 / 1893(0.8)$ & $13 / 1882(0.7)$ \\
\hline \multicolumn{4}{|l|}{ Coagulation } \\
\hline \multirow[t]{2}{*}{ Activated partial thromboplastin time (s) } & $<0.5 \times$ LLN and $>50 \%$ decrease from baseline & $0 / 1617(0.0)$ & $0 / 1642(0.0)$ \\
\hline & $>2 \times$ ULN and $>100 \%$ increase from baseline & $8 / 1617(0.5)$ & $18 / 1642(1.1)$ \\
\hline \multirow[t]{2}{*}{ International normalised ratio } & $<0.5 \times$ LLN and $>50 \%$ decrease from baseline & $0 / 1638(0.0)$ & $0 / 1663(0.0)$ \\
\hline & $>2 \times \mathrm{ULN}$ and $>100 \%$ increase from baseline & $24 / 1638(1.5)$ & $13 / 1663(0.8)$ \\
\hline \multirow[t]{2}{*}{ Prothrombin time (s) } & $<0.5 \times$ LLN and $>50 \%$ decrease from baseline & $0 / 1627(0.0)$ & $0 / 1652(0.0)$ \\
\hline & $>2 \times \mathrm{ULN}$ and $>100 \%$ increase from baseline & $24 / 1627(1.5)$ & $14 / 1652(0.8)$ \\
\hline
\end{tabular}

$A L P$ alkaline phosphatase, $A L T$ alanine aminotransferase, $A S T$ aspartate aminotransferase, $L L N$ lower limit of normal value, $m$ number of patients with both baseline and post-baseline values, $n$ number of patients with potentially clinically significant criteria, $N$ number of patients in treatment group, $U L N$ upper limit of normal 


\subsubsection{Severe Hypersensitivity}

Severe hypersensitivity was identified as an STOI based on the known ADRs of ceftazidime, which include anaphylaxis (including bronchospasm and/or hypotension), Stevens-Johnson syndrome, toxic epidermal necrolysis and erythema multiforme [23]. No AEs of anaphylaxis, StevensJohnson syndrome, toxic epidermal necrolysis or erythema multiforme were reported. One ceftazidime-avibactam patient had an unrelated $\mathrm{AE}$ of anaphylactic reaction that was temporally related to a vancomycin infusion and had negative rechallenges with ceftazidime-avibactam. Overall, the incidence of patients with at least one hypersensitivity AE was $8.3 \%$ for ceftazidime-avibactam \pm metronidazole and $7.5 \%$ for the comparator group (see Online Resource 5 in the ESM). Three patients with cIAI who received ceftazidime-avibactam + metronidazole had non-serious hypersensitivity AEs (rash pruritic, rash macular, and pruritus and urticaria, respectively) that were assessed by the investigator as causally related to study drug and resulted in discontinuation of study drug.

\subsubsection{Haematological Disorders}

The incidence of haematological disorders, identified by AEs, was $<4 \%$ across treatment groups (see Online Resource 5 [ESM]). The estimated Coombs seroconversion rate is based on data from 987 (48.8\%) patients in the phase II and III pool who had a baseline negative Coombs test and at least one follow-up test. Coombs seroconversion rates at any time up to the last visit for patients with a baseline negative result and at least one post-baseline positive result were $13.0 \%$ for ceftazidime-avibactam \pm metronidazole and $3.8 \%$ for comparators (Table 5). For the ceftazidime-avibactam \pm metronidazole group, the lowest percentage of Coombs seroconversion at any time up to the last visit was in patients with cUTI (6.3\%), and the highest was in patients with NP/VAP (21.4\%). Further analysis was conducted to include patients who had at least two post-baseline Coombs results, of which the first was negative. This was to include patients with a missing baseline value but with evidence of seroconversion. An additional 10 patients were identified, resulting in a frequency of $14.0 \%$ (138/987 patients). This was the most common potentially clinically significant haematological finding observed with ceftazidime-avibactam across all indications (cIAI 13.3\%; cUTI 6.3\%; NP/VAP $21.4 \%$ ). However, no patients with evidence of Coombs seroconversion had an AE or laboratory evidence of haemolytic anaemia.

\subsubsection{Renal Disorders}

The incidence of patients who developed a renal disorder AE was $1.6 \%$ for ceftazidime-avibactam \pm metronidazole and $1.2 \%$ for comparators (see Online Resource 5 [ESM]). No patient with renal impairment (estimated $\mathrm{CrCl} \leq 50 \mathrm{~mL} / \mathrm{min}$ ) was identified as having experienced an $\mathrm{AE}$ of neurological sequelae resulting from overexposure to ceftazidime-avibactam.

\subsection{Intrinsic and Extrinsic Factors}

Incidences of AEs (irrespective of relationship to study therapy), AEs leading to discontinuation of study drug (DAEs), SAEs and AEs of severe intensity for ceftazidime-avibactam \pm metronidazole and comparators assessed by age, gender, BMI, race, or renal function (intrinsic patient factors) are shown in Online Resources 6-11 (see ESM) and AEs by the extrinsic factor of geographic region are shown in Online Resource 11 (see ESM).

\subsection{Clinical Laboratory Assessments}

Potentially clinically significant laboratory changes compared with baseline are shown in Table 5. Prothrombin times $>2 \times \mathrm{ULN}$ and $>100 \%$ increase from baseline occurred in $1.5 \%$ and $0.8 \%$ of patients in the ceftazidime-avibactam \pm metronidazole and comparator groups, respectively, and an international normalised ratio (INR) of $>2 \times$ ULN and $>100 \%$ increase from baseline was found in $1.5 \%$ and $0.8 \%$ of patients, respectively (Table 5).

\subsection{Vital Signs and ECG Data}

Vital signs and ECG data were not pooled for the phase II and III studies. Changes in mean vital signs were reported individually for each study and were not considered clinically significant [16-22]. Supratherapeutic doses of ceftazidime-avibactam (3000 mg ceftazidime $+2000 \mathrm{mg}$ avibactam) did not result in a clinically meaningful increase in the Fridericia-corrected QT (QTcF) in a phase I, four-way crossover thorough QT study with moxifloxacin as positive control [9]. No new safety concerns for vital signs were noted in the individual phase II and III studies.

\subsection{Drug-Drug Interactions}

The following concomitant medications were selected for analysis of potential DDI: medications that have the ability 
to affect renal function; OAT1/OAT3 inhibitors; vitamin K antagonists (VKAs); chloramphenicol and sodium picosulphate. In patients who received a selected concomitant medication, AEs were assessed after the patient had received the first dose. Of note, such assessments can be confounded because observed AEs may be due to, or associated with, an underlying condition for which a concomitant medication would be indicated or may be associated with another ongoing concomitant medication. No evidence of DDIs between ceftazidime-avibactam \pm metronidazole and any of the selected concomitant medications was identified in these analyses (Table 6).

\section{Discussion}

The current pooled analysis included seven trials conducted in patients with cIAI, cUTI or NP, including VAP, and is representative of the patients for whom ceftazidime-avibactam will be prescribed [28, 29]. For an antibiotic, this pooled analysis comprises an adequate safety dataset (of 2024 patients treated with ceftazidime-avibactam \pm metronidazole from seven clinical trials), representative of the population for treatment (cIAI, cUTI and NP), and included 661 elderly patients (aged $\geq 65$ years). Comparator treatments represented standard therapies in the respective indications and were largely carbapenems. Across the trials, patients in each treatment arm could also receive concomitant antibiotics; however, no change to the safety profile of ceftazidime-avibactam was identified as a result of this. As the NP/VAP population had significant comorbidities and high APACHE II scores at baseline, they are considered reflective of adult patients with infections due to serious aerobic Gram-negative organisms with limited treatment options.

This comprehensive safety review was structured to reflect CIOMS Working Group IV recommendations [22]. In line with CONSORT guidance, safety data in the individual trials and in this pooled analysis were not subject to inferential statistical analysis/hypothesis testing [30]. As safety findings can be infrequent, it was not possible to fully assess the effect of intrinsic and extrinsic factors that might have an impact on safety in the individual studies. Based on ICH guidance [25], the frequencies and patterns of AEs across subpopulations according to age, sex, BMI, baseline renal function, race and geographical region were analysed descriptively for ceftazidime-avibactam and comparators. Specific evaluations based on the known safety profile of ceftazidime monotherapy and performed programmatically using Standardised MedDRA Queries included severe hepatotoxicity, CDAD, severe hypersensitivity, haematological disorders/bone marrow toxicity and renal disorders.

The analysis confirms that the safety profile of ceftazidime-avibactam and the most commonly reported AEs in the pooled analysis were consistent with the known safety profile for ceftazidime monotherapy [23]. The most frequent AEs included gastrointestinal symptoms and liver transaminase elevations, with no observed increases in the expected severity or frequency. The incidence of SAEs and AEs leading to discontinuation was low, and while numerically higher for ceftazidime-avibactam than the pooled comparator group, this difference was not large enough to be assessed as clinically impactful. Most SAEs in the ceftazidime-avibactam \pm metronidazole group were known ADRs of ceftazidime [23]. The remaining SAEs were likely attributable to the patients' underlying conditions and did not indicate any new safety findings. Common drug-related AEs for ceftazidime-avibactam \pm metronidazole comprised diarrhoea $(2.2 \%)$, headache $(1.0 \%)$, nausea $(1.0 \%)$ and increased ALT $(0.8 \%)$; all of which are expected ADRs for both ceftazidime and metronidazole $[23,31]$. Within each indication, the incidence of any AE up to the last study visit was balanced between treatment groups; across indications, the incidence was lowest in patients with cUTI and highest in patients with NP/VAP. This is to be expected, since patients with NP/ VAP tended to be older than those with cUTI or cIAI and were thus more likely to experience AEs [32]. In addition, patients with NP/VAP had an underlying comorbidity that had caused their original admission to hospital and higher baseline APACHE II scores than those with cIAI (APACHE II data were not recorded for patients with cUTI), consistent with more severe baseline disease, and thus a greater expected AE burden.

Avibactam has linear pharmacokinetics, with minimal metabolism, high bioavailability and low potential for protein binding and drug-drug interactions [6-15]. There are currently no identified human receptors for avibactam, and the only ADR identified for avibactam to date, based on animal data, is phlebitis; however, no increase in the severity or frequency of phlebitis in the clinical development programme was observed, compared with that expected for ceftazidime monotherapy [23].

STOIs based on the known safety profile of ceftazidime included liver disorders, diarrhoea including CDAD, severe hypersensitivity including anaphylaxis, haematological disorders and renal disorders. Overall, the incidences of AEs related to liver disorders, diarrhoea, severe hypersensitivity or renal disorders for patients taking ceftazidime-avibactam \pm metronidazole were similar to those expected for ceftazidime monotherapy [23].

No meaningful trends or pattern of changes in haematological or clinical chemistry parameters were identified. Antibiotics that may be associated with seroconversion to a positive Coombs test and, very rarely, a subsequent diagnosis of haemolytic anaemia include penicillin and other $\beta$-lactams [33]. A higher incidence of positive Coombs test in the ceftazidime-avibactam \pm metronidazole group versus 
the expected labelled frequency for ceftazidime [23] was observed. However, the ceftazidime monotherapy development programme encompassed more infection types than the ceftazidime-avibactam programme, and the individual frequencies of a positive Coombs test with ceftazidime monotherapy in each infection type are not publicly available. Therefore, it is not possible to assess whether this increase is also seen for ceftazidime monotherapy when considering only the three indications studied in the ceftazidime-avibactam programme. The sensitivity of the assays used to calculate the frequency of a positive Coombs test in the ceftazidime-avibactam trials may also differ to those used in the original ceftazidime monotherapy trials. As positive Coombs test results in the pooled safety dataset were not associated with reported AEs of haemolysis, nor any related evidence in clinical haematology assessments, this finding is not considered to be clinically significant. This will continue to be monitored in ongoing post-marketing surveillance.

The frequency of potentially clinically significant laboratory changes was low across indication groups. Although hypokalaemia was reported more frequently as an $\mathrm{AE}$ in patients with NP than patients with cIAI and cUTI, it was not confirmed by analysis of actual change in serum potassium values from baseline to individual study day, end of treatment, test-of-cure or last visit, and these events were concluded to be associated with the patients' underlying and concomitant medical conditions.

There was no evidence that the safety profile of ceftazidime-avibactam is influenced by intrinsic factors (such as race, age, gender, BMI and renal function), or extrinsic factors (such as geographic region), and there was no evidence of DDIs with selected concomitant medications; however, these analyses are limited by the denominator of patients in some subgroups. As both ceftazidime and avibactam are primarily cleared by the kidney, dosage adjustments are required in patients with moderate or severe renal impairment (estimated $\mathrm{CrCl}<50 \mathrm{~mL} / \mathrm{min}$ ). There were no clinically important safety concerns observed in patients by subgroups of renal function, although again denominators were small in some subgroups, particularly severe renal impairment. There was no evidence that the safety profile was affected by extreme age, and this pooled analysis comprised an adequate dataset in this group (approximately $16 \%$ of patients were aged $\geq 75$ to $\leq 90$ years).

A recent systematic review and meta-analysis evaluated the efficacy and top-line safety of ceftazidime-avibactam based on 3988 patients in the phase II-III clinical trials, and was generally consistent with the results of the current pooled analysis [26]. Our analysis in all 4050 patients in the pooled safety population provides a more comprehensive overview of safety using pre-specified programmed safety analysis, treatment-emergent AEs and patient-level data, rather than only the summaries available in the published literature, and with respect to REPROVE, included a subpopulation of moderate and severely impaired renal function at baseline, which was analysed separately in the study following a protocol amendment and change to dosage regimens [22]. While meta-analysis has important applications, particularly for analysing efficacy outcomes of different interventions across multiple trials, its use for assessing safety from pooled clinical trial data has various methodological challenges and limitations [34-38]. In particular, considering heterogeneous treatments as a single comparator group for meta-analysis of safety outcomes is potentially misleading and a challenge for interpretation. In the context of the current analysis, ceftazidime-avibactam (a cephalosporin $\beta$-lactam antibiotic) \pm metronidazole was compared with a heterogenous group of comparator treatments consisting mainly of carbapenem $\beta$-lactam antibiotics, which have similar, but not identical, safety and tolerability profiles to cephalosporins $[39,40]$. As such, minor differences in safety outcomes between ceftazidime-avibactam

Table 6 Adverse events up to the last study visit in patients receiving selected concomitant medications with potential for drug-drug interactions (pooled safety population)

\begin{tabular}{lccccc}
\hline $\begin{array}{l}\text { Patients with any AE with/without concurrent } \\
\text { administration, } n(\%)\end{array}$ & $\begin{array}{l}\text { Ceftazidime-avibactam } \pm \text { metronidazole } \\
(N=2024)\end{array}$ & & Comparator $(N=2026)$ \\
\cline { 2 - 3 } \cline { 5 - 6 } & $\begin{array}{l}\text { With concurrent } \\
\text { medication }\end{array}$ & $\begin{array}{l}\text { Without concurrent } \\
\text { medication }\end{array}$ & & $\begin{array}{l}\text { With concurrent } \\
\text { medication }\end{array}$ & $\begin{array}{l}\text { Without concurrent } \\
\text { medication }\end{array}$ \\
\hline Drugs with ability to affect renal function & $143 / 265(54.0)$ & $236 / 588(40.1)$ & & $168 / 284(59.2)$ & $199 / 578(34.4)$ \\
Vitamin K antagonists & $5 / 10(50.0)$ & $386 / 844(45.7)$ & & $7 / 13(53.8)$ & $368 / 848(43.4)$ \\
Chloramphenicol & $4 / 4(100.0)$ & $394 / 853(46.2)$ & & $1 / 2(50.0)$ & $378 / 860(44.0)$ \\
OAT1 and OAT3 inhibitors & $26 / 58(44.8)$ & $364 / 793(45.9)$ & & $29 / 65(44.6)$ & $346 / 795(43.5)$ \\
Sodium picosulfate & $1 / 1(100.0)$ & $397 / 856(46.4)$ & & $1 / 2(50.0)$ & $380 / 861(44.1)$ \\
\hline
\end{tabular}

Percentages are based on the total number of patients in the subgroup and treatment group $(N)$. For patients who received a selected concomitant medication, AEs were assessed after the patient had received the first dose of concomitant medication

$A E$ adverse event 
and the pooled comparator group (which were evident in our descriptive analyses) would be expected based on the different drug classes alone, and particularly when considering the addition of metronidazole to ceftazidime-avibactam in the cIAI population.

In our pooled analysis, a consistent pattern of safety data was demonstrated for ceftazidime-avibactam \pm metronidazole and the comparator agents within infection types. The higher incidence of SAEs and all-cause mortality in the NP/VAP population reflects the fact that the phase III NP study, REPROVE [22], enrolled patients who were older and tended to be sicker (i.e. those who were hospitalised for another reason and then acquired pneumonia) at baseline compared with the other studies. Similar to the current analysis, mortality rates reported in the literature are highest for NP (up to $62 \%$ ), followed by cIAI (ranging from $<25 \%$ to $>50 \%$ depending on the location of the infection and the form of peritonitis involved) and cUTI (20-40\%) [41-43].

A limitation of analysing pooled data is the potential for differences in baseline characteristics of the individual study populations and in patients with different infection types. Our analyses were limited by the heterogeneous treatments given to the comparator group and the potential for bias in inferential testing of safety outcomes in the clinical studies (which were primarily designed to assess efficacy). Another specific limitation of the current analysis is that subtle differences in the safety profile between ceftazidime-avibactam and historical data for ceftazidime monotherapy may be difficult to identify because very large patient populations would be required $[44,45]$. The studies included in this analysis did not use ceftazidime as an active control, so no direct comparison with ceftazidime-avibactam was possible. However, no clinically significant findings were made after comparison of safety parameters in the ceftazidime-avibactam studies with the safety profile detailed in the ceftazidime prescribing information [23]. These observations support the view that the safety profiles of ceftazidime-avibactam and ceftazidime are clinically similar.

Although the analysis included a heterogeneous patient population from several trials in different infection types, the patients had many common eligibility criteria and data were collected and analysed in a standardised manner. The comparable safety profiles of ceftazidime-avibactam and comparators in patients with cIAI, cUTI and NP demonstrated consistency of results within each patient population, when the patients' respective underlying medical conditions are considered.

\section{Conclusions}

This pooled analysis of the ceftazidime-avibactam phase II and III clinical trial programme confirms that the current emerging safety profile of ceftazidime-avibactam is similar to the known profile of ceftazidime monotherapy, as defined following decades of post-marketing experience, and that the addition of avibactam does not result in clinically relevant differences. These data form the basis of the safety text approved by the regulatory agencies for ceftazidime-avibactam $[28,29]$. Continued active post-marketing surveillance will further define the safety profile of ceftazidime-avibactam in real-world practice. Overall, this analysis supports the use of ceftazidime-avibactam as a treatment option in adults with cUTI, cIAI and NP, including VAP.

Acknowledgements The authors would like to thank the patients, their families and all investigators involved in the studies on ceftazidimeavibactam. Thanks also to Tom Gibbons and Dheeraj Rupani for programming support with the preparation of the pooled analysis.

\section{Compliance with Ethical Standards}

Funding The studies NXL104-2001 and NXL104-2002 were sponsored by Novexel, and rights were subsequently acquired by AstraZeneca; RECLAIM 1 and 2 (NCT01499290); RECLAIM 3 (NCT01726023); REPRISE (NCT01644643); RECAPTURE 1 and 2 (NCT01595438 and NCT01599806); and REPROVE (NCT01808092) were originally sponsored by AstraZeneca and are now sponsored by Pfizer. AstraZeneca's rights to ceftazidime-avibactam were acquired by Pfizer in December 2016. Medical writing support was provided by Jennifer Shepherd, PhD, Mark Waterlow BSc, ISMPP CMPP, and Valerie Moss, PhD, ISMPP CMPP, of Prime, Knutsford, Cheshire and was funded by AstraZeneca and Pfizer. Ultimate responsibility for opinions, conclusions and data interpretation lies with the authors.

Conflict of interest All authors except K.Y. were employees of AstraZeneca at the time of the completion of the ceftazidime-avibactam phase III studies and/or during preparation of the manuscript. P.N., J.W.C., H.B., D.W. and A.W. are current or were shareholders in AstraZeneca during the conduct of the ceftazidime-avibactam phase III studies and/or during preparation of the manuscript. K.Y. was a contractor to AstraZeneca at the time of the studies and during manuscript development. K.C. and J.W.C. are currently employees of Pfizer.

Ethical approval All studies were conducted in compliance with the Declaration of Helsinki and/or the International Conference on Harmonization Good Clinical Practice Guidelines. All study protocols (NXL104-2001, NXL104-2002, NCT01499290, NCT01726023, NCT01644643, NCT01595438, NCT01599806 and NCT01808092) were approved by relevant Institutional Review Boards and/or Independent Ethics Committees. All patients (or their representatives) provided written informed consent. 
Data sharing Upon request, and subject to certain criteria, conditions and exceptions (see https://www.pfizer.com/science/clinical-trials/trial -data-and-results for more information), Pfizer will provide access to individual de-identified participant data from Pfizer-sponsored global interventional clinical studies conducted for medicines, vaccines and medical devices (1) for indications that have been approved in the US and/or EU or (2) in programmes that have been terminated (i.e., development for all indications has been discontinued). Pfizer will also consider requests for the protocol, data dictionary, and statistical analysis plan. Data may be requested from Pfizer trials 24 months after study completion. The de-identified participant data will be made available to researchers whose proposals meet the research criteria and other conditions, and for which an exception does not apply, via a secure portal. To gain access, data requestors must enter into a data access agreement with Pfizer.

Open Access This article is licensed under a Creative Commons Attribution-NonCommercial 4.0 International License, which permits any non-commercial use, sharing, adaptation, distribution and reproduction in any medium or format, as long as you give appropriate credit to the original author(s) and the source, provide a link to the Creative Commons licence, and indicate if changes were made. The images or other third party material in this article are included in the article's Creative Commons licence, unless indicated otherwise in a credit line to the material. If material is not included in the article's Creative Commons licence and your intended use is not permitted by statutory regulation or exceeds the permitted use, you will need to obtain permission directly from the copyright holder. To view a copy of this licence, visit http://creativecommons.org/licenses/by-nc/4.0/.

\section{References}

1. Taneja N, Kaur H. Insights into Newer Antimicrobial Agents Against Gram-negative Bacteria. Microbiol Insights. 2016;9:9-19.

2. Boucher HW, Talbot GH, Benjamin DK Jr, Bradley J, Guidos RJ, Jones RN, et al. $10 \times$ '20 Progress-development of new drugs active against gram-negative bacilli: an update from the Infectious Diseases Society of America. Clin Infect Dis. 2013;56(12):1685-94.

3. Boucher HW, Talbot GH, Bradley JS, Edwards JE, Gilbert D, Rice LB, et al. Bad bugs, no drugs: no ESKAPE! An update from the Infectious Diseases Society of America. Clin Infect Dis. 2009;48(1):1-12.

4. Munoz-Price LS, Poirel L, Bonomo RA, Schwaber MJ, Daikos GL, Cormican M, et al. Clinical epidemiology of the global expansion of Klebsiella pneumoniae carbapenemases. Lancet Infect Dis. 2013;13(9):785-96.

5. Keepers TR, Gomez M, Celeri C, Nichols WW, Krause KM. Bactericidal activity, absence of serum effect, and time-kill kinetics of ceftazidime-avibactam against beta-lactamase-producing Enterobacteriaceae and Pseudomonas aeruginosa. Antimicrob Agents Chemother. 2014;58(9):5297-305.

6. Merdjan H, Tarral A, Das S, Li J. Phase 1 study assessing the pharmacokinetic profile and safety of avibactam in patients with renal impairment. J Clin Pharmacol. 2017;57(2):211-8.

7. Tarral A, Merdjan H. Effect of age and sex on the pharmacokinetics and safety of avibactam in healthy volunteers. Clin Ther. 2015;37(4):877-86.

8. Merdjan H, Rangaraju M, Tarral A. Safety and pharmacokinetics of single and multiple ascending doses of avibactam alone and in combination with ceftazidime in healthy male volunteers: results of two randomized, placebo-controlled studies. Clin Drug Investig. 2015;35(5):307-17.

9. Das S, Armstrong J, Mathews D, Li J, Edeki T. Randomized, placebo-controlled study to assess the impact on QT/QTc interval of supratherapeutic doses of ceftazidime-avibactam or ceftaroline fosamil-avibactam. J Clin Pharmacol. 2014;54(3):331-40.

10. Vishwanathan K, Mair S, Gupta A, Atherton J, Clarkson-Jones J, Edeki T, et al. Assessment of the mass balance recovery and metabolite profile of avibactam in humans and in vitro drug-drug interaction potential. Drug Metab Dispos. 2014;42(5):932-42.

11. Nicolau D, Siew L, Armstrong J, Li J, Edeki T, Learoyd M, et al. Phase 1 study assessing the steady-state concentration of ceftazidime and avibactam in plasma and epithelial lining fluid following two dosing regimens. J Antimicrob Chemother. 2015;70(10):2862-9.

12. Tominaga N, Edeki T, Li J, Learoyd M, Bouw MR, Das S. Phase I study assessing the safety, tolerability, and pharmacokinetics of avibactam and ceftazidime-avibactam in healthy Japanese volunteers. J Infect Chemother. 2015;21(8):551-8.

13. Das S, Li J, Armstrong J, Learoyd M, Edeki T. Randomized pharmacokinetic and drug-drug interaction studies of ceftazidime, avibactam, and metronidazole in healthy subjects. Pharmacol Res Perspect. 2015;3(5):e00172.

14. Li J, Learoyd M, Qiu F, Zhu L, Edeki T. A randomized, phase I study to assess the safety, tolerability and pharmacokinetics of ceftazidime-avibactam in healthy chinese subjects. Clin Drug Investig. 2016;36(2):119-26.

15. Rashid MU, Rosenborg S, Panagiotidis G, Lofdal KS, Weintraub A, Nord CE. Ecological effect of ceftazidime/avibactam on the normal human intestinal microbiota. Int J Antimicrob Agents. 2015;46(1):60-5.

16. Vazquez JA, Gonzalez Patzan LD, Stricklin D, Duttaroy DD, Kreidly Z, Lipka J, et al. Efficacy and safety of ceftazidime-avibactam versus imipenem-cilastatin in the treatment of complicated urinary tract infections, including acute pyelonephritis, in hospitalized adults: results of a prospective, investigator-blinded, randomized study. Curr Med Res Opin. 2012;28(12):1921-31.

17. Lucasti C, Popescu I, Ramesh MK, Lipka J, Sable C. Comparative study of the efficacy and safety of ceftazidime/avibactam plus metronidazole versus meropenem in the treatment of complicated intra-abdominal infections in hospitalized adults: results of a randomized, double-blind, Phase II trial. J Antimicrob Chemother. 2013;68(5):1183-92.

18. Wagenlehner FM, Sobel JD, Newell P, Armstrong J, Huang X, Stone GG, et al. Ceftazidime-avibactam versus doripenem for the treatment of complicated urinary tract infections, including acute pyelonephritis: RECAPTURE, a phase 3 randomized trial program. Clin Infect Dis. 2016;63(6):754-62.

19. Carmeli Y, Armstrong J, Laud PJ, Newell P, Stone G, Wardman A, et al. Ceftazidime-avibactam or best available therapy in patients with ceftazidime-resistant Enterobacteriaceae and Pseudomonas aeruginosa complicated urinary tract infections or complicated intra-abdominal infections (REPRISE): a randomised, pathogen-directed, phase 3 study. Lancet Infect Dis. 2016;16(6):661-73.

20. Mazuski JE, Gasink LB, Armstrong J, Broadhurst H, Stone GG, Rank D, et al. Efficacy and safety of ceftazidime-avibactam plus metronidazole versus meropenem in the treatment of complicated intra-abdominal infection: results from a randomized, controlled, double-blind, phase 3 program. Clin Infect Dis. 2016;62(11):1380-9.

21. Qin X, Tran BG, Kim MJ, Wang L, Nguyen DA, Chen Q, et al. A randomised, double-blind, phase 3 study comparing the efficacy and safety of ceftazidime/avibactam plus metronidazole versus meropenem for complicated intra-abdominal 
infections in hospitalised adults in Asia. Int $\mathbf{J}$ Antimicrob Agents. 2017;49(5):579-88.

22. Torres A, Zhong N, Pachl J, Timsit JF, Kollef M, Chen Z, et al. Ceftazidime-avibactam versus meropenem in nosocomial pneumonia, including ventilator-associated pneumonia (REPROVE): a randomised, double-blind, phase 3 non-inferiority trial. Lancet Infect Dis. 2018;18(3):285-95.

23. GlaxoSmithKline. Fortum $2 \mathrm{~g}$ powder for solution for injection or infusion. 2016. https://www.medicines.org.uk/emc/produ ct/1774/smpc. Accessed 4 Feb 2020.

24. Council for International Organizations of Medical Sciences (CIOMS). Management of Safety Information from Clinical Trials: Report of CIOMS Working Group VI. 2005. https:// cioms.ch/wp-content/uploads/2017/01/Mgment_Safety_Info. pdf. Accessed 4 Feb 2020

25. International Council for Harmonisation of Technical Requirements for Pharmaceuticals for Human Use. Ethnic factors in the acceptability of foreign clinical data E5(R1). 1998. https://datab ase.ich.org/sites/default/files/E5_R1_Guideline.pdf. Accessed $4 \mathrm{Feb} 2020$.

26. Sternbach N, Leibovici Weissman Y, Avni T, Yahav D. Efficacy and safety of ceftazidime/avibactam: a systematic review and meta-analysis. J Antimicrob Chemother. 2018;73(8):2021-9.

27. European Medicines Agency. Summary of risk management plan for CAZ-AVI. 2018. https://www.ema.europa.eu/en/docum ents/rmp-summary/zavicefta-epar-risk-management-plansummary_en.pdf. Accessed 4 Feb 2020.

28. Allergan. AVYCAZ (ceftazidime and avibactam) for injection, for intravenous use. 2019. https://www.allergan.com/assets/pdf/ avycaz_pi. Accessed 6 Feb 2020.

29. Pfizer. Summary of Product Characteristics: Zavicefta $2 \mathrm{~g} / 0.5 \mathrm{~g}$ powder for concentrate for solution for infusion. 2020. http:// www.ema.europa.eu/docs/en_GB/document_library/EPAR_-_ Product_Information/human/004027/WC500210234.pdf. Accessed 6 Feb 2020

30. Ioannidis JP, Evans SJ, Gotzsche PC, O’Neill RT, Altman DG, Schulz K, et al. Better reporting of harms in randomized trials: an extension of the CONSORT statement. Ann Intern Med. 2004;141(10):781-8.
31. Baxter Healthcare Ltd. Metronidazole $500 \mathrm{mg} / 100 \mathrm{ml}$ intravenous infusion. 2017. https://www.medicines.org.uk/emc/product/1842/ smpc. Accessed 4 Febr 2020.

32. Sari AB, Cracknell A, Sheldon TA. Incidence, preventability and consequences of adverse events in older people: results of a retrospective case-note review. Age Ageing. 2008;37(3):265-9.

33. Arndt PA, Garratty G. The changing spectrum of drug-induced immune hemolytic anemia. Semin Hematol. 2005;42(3):137-44.

34. Bigger JT. Issues in subgroup analyses and meta-analyses of clinical trials. J Cardiovasc Electrophysiol. 2003;14(9 Suppl):S6-8.

35. Kuss O. Statistical methods for meta-analyses including information from studies without any events-add nothing to nothing and succeed nevertheless. Stat Med. 2015;34(7):1097-116.

36. Siddiqui O. Statistical methods to analyze adverse events data of randomized clinical trials. J Biopharm Stat. 2009;19(5):889-99.

37. Bradburn MJ, Deeks JJ, Berlin JA, Russell Localio A. Much ado about nothing: a comparison of the performance of meta-analytical methods with rare events. Stat Med. 2007;26(1):53-77.

38. Wittes J, Crowe B, Chuang-Stein C, Guettner A, Hall D, Jiang Q, et al. The fda's final rule on expedited safety reporting: statistical considerations. Stat Biopharm Res. 2015;7(3):174-90.

39. Carbapenems. In: Aronson JK, editor. Meyler's Side Effects of Drugs. Oxford: Elsevier; 2016. p. 99-102.

40. Cephalosporins. In: Aronson JK, editor. Meyler's Side Effects of Drugs. Oxford: Elsevier; 2016. p. 197-215.

41. Chan JF, Yuen KY. A new ASPECT for complicated urinary tract infections. Lancet. 2015;385(9981):1920-2.

42. Herzog T, Chromik AM, Uhl W. Treatment of complicated intraabdominal infections in the era of multi-drug resistant bacteria. Eur J Med Res. 2010;15(12):525-32.

43. Rotstein C, Evans G, Born A, Grossman R, Light RB, Magder S, et al. Clinical practice guidelines for hospital-acquired pneumonia and ventilator-associated pneumonia in adults. Can J Infect Dis Med Microbiol. 2008;19(1):19-53.

44. Lewis J. Post-marketing surveillance: how many patients? Trends Pharmacol Sci. 1981;2:93-4.

45. Joelson S, Joelson IB, Wallander MA. Geographical variation in adverse event reporting rates in clinical trials. Pharmacoepidemiol Drug Saf. 1997;6(Suppl 3):S31-5. 